\title{
Inhalts-Verzeichniss des ersten Bandes.
}

Seite

Vorwort zur Deutschen Bearbeitung

Prolegomena.

Einleitung . . . . . . . . . . . . . . . . . . . . 1

Erste Abthellung. Von der chirurgischen Diagnostik . . . . . . . 4

Erstes Capitel. Von der Anwendung der Sinne bei der chirurgischen Diagnostik . . . . . . . . . . . . . . . . . . 5

I. Gesichtssinn. . . . . . . . . . . . . . . . . . 5

II. Tastsinn . . . . . . . . . . . . . . . . . . . 12

III. Gelörssinn . . . . . . . . . . . . . . . . . . 15

IV. Geruchssinn . . . . . . . . . . . . . . . . . . 19

v. Geschmackssinn . . . . . . . . . . . . . . . . 20

Zweites Capitel. Von den aus den sinnlichen Wahrnebmungen zu ziehenden Schlüssen . . . . . . . . . . . . . . . 20

Zwelte Abthellung. Von den der Chirurgie eigenthümlichen Heilmitteln.

Erster Abschnitt. Von den chirurgischen Operationen im Allgemeinen 23

I. Von den Operationsmethoden und Operations-Verfalren. . . . 24

II. Regelmässige Operationen. Ungewöhnliche Operationen . . . . 25

III. Wahl des Ortes und der Zeit . . . . . . . . . . . . 25

IV. Operationen, die man unterlassen sollte. Nothwendige und nützliche Operationen . . . . . . . . . . . . . . . . 26

v. Vertheilung einer Operation auf verschiedene Zeiten . . . . 28

VI. Verfahren vor, wälrend und nach der 0peration . . . . . . 31 Sicherung gegen Blutung . . . . . . . . . . . . . . 36

Aufhebung der Empfindlichkeit . . . . . . . . . . . . $\mathbf{4 0}$

vII. Zufälle bei Operationen . . . . . . . . . . . . . . 49

A) Obnmacht . . . . . . . . . . . . . . . . . . . 51 
B) Krämple . . . . . . . . . . 52

C) Blutung . . . . . . . . . . . . . . . . . . . 53

D) Eintritt von Luft in die Venen . . . . . . . . . . 54

Zweiter Abschnitt. Von den Verbänden.

I. Regeln für das Verbinden . . . . . . . . . . . . . 62

II. Instrumente zum Verbinden (Verbandzeug) . . . . . . . . 65

III. Verbandgegenstände.

A) Charpie und deren Surrogate . . . . . . . . . . . . 71

B) Aeusserliche Arzneimittel . . . . . . . . . . . . . . 75

Pulver, Pnaster, Salben, Breiumschläge, Flüssige Formen (Anwendung des Wassers, Klystiere), Elastisch nüssige Formen.

C) Compressen . . . . . . . . . . . . . . . . . . . 85

D) Binden . . . . . . . . . . . . . . . . . . . 87

Dritter Abschnitt. Von den Elementar-Operationen . . . . . 93

Erstes Capitel. Von der Trennung.

I. Von der Art, die scbneidenden Instrumente zu halten.

A) Von den verschiedenen Arten, das Messer zu balten . . . 94

B) Von der Haltung der Scheere. . . . . . . . . . . 97

II. Incisionen . . . . . . . . . . . . . . 98

A) Von Aussen nach lnnen . . . . . . . . . . . 100

B) Von Innen nach Aussen . . . . . . . . . . . . 102

C) Subcutane Einschnitte . . . . . . . . . . . . . 104

III. Vom Präpariren . . . . . . . . . . . . . . 106

IV. Von der Punction . . . . . . . . . . . . . . 106

A) Mit der Acupuncturnadel . . . . . . . . . . . . 107

B) Mit dem Troiquart . . . . . . . . . . . . 107

c) Mit dem Messer . . . . . . . . . . . . . . . 108

v. Vom Aetzen . . . . . . . . . . . . . . . . . 109

A) Cauterium actuale . . . . . . . . . . . . . . 109

в) Moxa . . . . . . . . . . . . . . . . . . 111

C) Cauterium polentiale . . . . . . . . . . . . . 112

Zweites Capitel. Von der Vereinigung . . . . . . . . 115

I. Lage . . . . . . . . . . . . . . . . . 115

II. Vereinigende Binden . . . . . . . . . . . . 116

III. Heftplaster und andere Klebstoffe . . . . . . . . . 118

Jv. Nähte . . . . . . . . . . . . . . . . . . 119

1) Knopfnaht . . . . . . . . . . . . . . . . . 120

2) Umschlungene Naht . . . . . . . . . . . . . 121

3) Zapfennaht . . . . . . . . . . . . . . . . . . . 122

Allgemeine Regeln für die Anlegung der Năhte . . . . . 122

Von dem Ausziehen der Nähte . . . . . . . . . 123 
vierter Absclunitt. Von den gewöhnlichen kleinen Operationen oder der sogenannten kleinen Clirurgie. . . . . . . . . 124

Erstes Capitel. Von den Blutentziehungen . . . . . . . . 124

I. Vom Aderlass . . . . . . . . . . . . . . . . 124

A) Am Arme . . . . . . . . . . . . . . . . . 126

B) Am Fusse . . . . . . . . . . . . . . . . . 135

c) Am Halse . . . . . . . . . . . . . . . . . 136

II. Von der Arteriotomie . . . . . . . . . . . . . 137

III. Von den capillaren oder örtlichen Blutentziehungen . . . . 139

A) Blutegel . . . . . . . . . . . . . . . . . 139

B) Scarificationen . . . . . . . . . . . . . . . 141

C) Schröplkōple . . . . . . . . . . . . . . . . 142

blutige . . . . . . . . . . . . . . . . 112

trockene . . . . . . . . . . . . . . . . . 144

Zweites Capitel. Von den Hautreizen und Ableitungsmitteln . 145

I. Senfteig . . . . . . . . . . . . . . . . . . 145

II. Blasenbildung . . . . . . . . . . . . . . 146

III. Fontanelle . . . . . . . . . . . . . . . . . 148

IV. Haarseil . . . . . . . . . . . . . . . . 151

Drittes Capitel. Von dem Impfen der Kuhpocken. . . . . 152

\section{Erstes Bucb.}

Von den chirurgischen Krankheiten im Allgemeinen.

Eroter Abschnitt. Von der Entzündung und ibren Ausgängeb.

Erstes Capitel. Von der Entzïndung im Allgemeinen . . . . . 157

Zweites Capitel. Von der Verbrennung . . . . . . . . . 173

Drittes Capitel. Von der Erfrierung . . . . . . . . . . . 184

Viertes Capitel. Von der Eiterung und der Abscessbildung . . . . 188

I. Von der Eiterung . . . . . . . . . . . . . . . . 188

A) Vom Eiter . . . . . . . . . . . . . . . . . . 188

B) Von der Bildung des Eiters . . . . . . . . . . . . 193

C) Von der Wirkung der Eiterung auf den übrigen Körper . . . 195

Il. Von dem Abscess im Allgemeinen . . . . . . . . . . . 197

Operationen zur Beseitigung der Abscesse . . . . . . . . . 209

1) Heisse oder phlegmonöse Abscesse . . . . . . . . . 212

2) Kalte Abscesse, Lymph-Abscesse . . . . . . . . . . 213

Fünftes Capitel. Vorn Brande... . . . . . . . . . . . 217

Sechstes Capitel. Von dem Brande durch anhaltenden Druck, dem

brandigen Aufliegen . . . . . . . . . . . . 229 
Seite

Siebentes Capitel. Vom Hospitalbrande . . . . . . . . . . 232

Formen und Verlauf des Hospitalbrandes . . . . . . . . . 233

Ursachen und Theorien über die Wirkungsweise derselben . . . . 235

Achtes Capitel. Vom Milzbrande . . . . . . . . . . . 242

I. Milzbrand-Carbunkel . . . . . . . . . . . . . . . 242

A) Bei den Thieren . . . . . . . . . . . . . . , 242

B) Beim Menschen . . . . . . . . . . . . . . , 244

II. Pustula mallgna . . . . . . . . . . . . . . . , 247

Neuntes Capitel. Von der Verschwärung . . . . . . . . . - 253

I. Vom Geschwïr . . . . . . . . . . . . . . . . - 257

Verschiedenheiten der Geschwüre . . . . . . . . . . , 258

II. Von den Fisteln . . . . . . . . . . . . . . . . , 264

Zwelter Abschnitt. Von den Verletzungen . . . . . . . . . . 269

Erstes Capitel. Trennungen der Continuität und Contiguität . . . 271

Zweites Capitel. Von den Wunden im Allgemeinen und den Schnittwunden im Besonderen . . . . . . . . . . . . . . 275

Drittes Capitel. Von den Stichwunden . . . . . . . . . . 279

Viertes Capitel. Von den gerissenen und gequetschten Wunden . - 282

A) Subcutane Zerreissungen. - Quetscliungen, Contusionen . . 282

B) Wunden durch Zerreissung mit Trennung der Haut. Gequetschte, zerrissene und Bisswunden . . . . . . . . . . . 285

Fünftes Capitel. Von den Zerschmetterungen und den Schusswunden. 288

Sechstes Capitel. Von den Störungen des Allgemeinbefindens bei Wunden und deren Behandlung . . . . . . . . . . . . . 305

Siebentes Capitel. Von den vergifteten Wunden . . . . . . . 308

1) Uebertragung der eigentlichen Gifte . . . . . . . . . . 308

2) Einimpfung normaler giftiger Secrete . . . . . . . . . 311

A) Insectenstiche . . . . . . . . . . . . . . . . 311

B) Schlangenbiss . . . . . . . . . . . . . . . . 311

3) Einimpfung von krankhaft veränderten Secreten . . . . . . $\mathbf{3 1 5}$

a) Wuthgift . . . . . . . . . . . . . . . . . 315

b) Rotzkrankheit . . . . . . . . . . . . . . . . 324

Bei den Einhufern . . . . . . . . . . . . . . 324

Beim Menschen . . . . . . . . . . . . . . . 325

Achtes Capitel. Von den fremden Körpern . . . . . . . . . . 328

Verschiedenlıeiten der fremden Körper . . . . . . . . . . . 328

Erscheinungen, welche die fremden Körper veranlassen . . . . . 329

Behandlung der fremden Körper . . . . . . . . . . . . . 333

Von der Ausfülirung der Exärese . . . . . . . . . . . . . 336

Dritter Abschnitt. Von den Missbildungen . . . . . . . . . . 337

Erstes Capitel. Von den Missbildungen im Allgemeinen . . . . . 337

Eintheilung der Missbildungen . . . . . . . . . . . . 341 
Zweites Capitel. Von den Defecten

Operative Plastik, plastische Operationen, plastische Chirurgie. . . 350

Drittes Capitel. Von den Verschmelzungen, Verwachsungen, dem Verschlusse normaler 0effnungen . . . . . . . . . . . . 362

Viertes Capitel. Von den Spaltbildungen . . . . . ... . 364

Fünftes Capitel. Von der Hypertrophie und der Ueberzahl einzelner Theile . . . . . . . . . . . . . . . . . . . 367

Trennung der Doppelmissbildungen . . . . . . . . . . . 368

Sechstes Capitel. Lageveränderungen. . . . . . . . . . . 369

Siebentes Capitel. Von den Verkrümmungen . . . . . . . 372

Behandlung der Verkrümmungen . . . . . . . . . . . 378

Vierter Abschnitt. Von den organisirten Neubildungen . . . . . . 382

Erstes Capitel. Einleitung und allgemeine Uebersicht . . . . . 382

Entstelung der Neubildungen . . . . . . . . . . . . . 384

Verschiedenheiten der Neubildungen . . . . . . . . . . . 387

Verlauf - $\quad-\quad$. . . . . . . . . . . 391

Diagnostik $\quad . \quad$ - $\quad . \quad . \quad . \quad . \quad . \quad . \quad . \quad . \quad . \quad .392$

Behandlung - $\quad-\quad$. . . . . . . . . . . . 396

Zweites Capitel. Pathologische Neubildung von Fettgewebe (Fettgescliwulst, Lipoma) . . . . . . . . . . . . : . . 407-

Drittes Capitel. Neubildung von Bindegewebe . . . . . . . 417

A. Narbengewebe . . . . . . . . . . . . . . . . . 418

1) Fehlerhafte Beschaffenheit der Narben . . . . . . . . 119

2) Formfehler durch Narben . . . . . . . . . . . . . 420

3) Krankheiten der Narben . . . . . . . . . . . . 421

B. Bindegewebsgeschwïlste oder Fosergeschwülste . . . . . . 422

Viertes Capitel. Speckgeschwulst . . . . . . . . . . . . 431

Fünftes Capitel. Muskelfleischgeschwulst. . . . . . . . . . 434

Sechstes Capitel. Polypen . . . . . . . . . . . . 436

Siebentes Capitel. Neubildung von Knorpelgewebe (Enchondrom). . 441

Achtes Capitel. Neubildung von Knochengewebe. Knochengeschwïlste 449

Neuntes Capitel. Cysten, Balggeschwülste . . . . . . . . . 451

Zehntes Capitel. Krebs . . . . . . . . . . . . . . . 470

Entwickelung und Metamorphosen des Krebsgewebes. . . . . . 471

Symptome, Verlauf, Ausgänge . . . . . . . . . . . . . 483

Diagnose. . . . . . . . . . . . . . . . . . . . 487

Aetiologie . . . . . . . . . . . . . . . . . . 488

Behandlung . . . . . . . . . . . . . . . . . . 490

Verschiedene Arten der Krebsgeschwïlste:

I. Epithelial- oder Epidermidalkrebs . . . . . . . . . 492

II. Faserkrebs . . . . . . . . . . . . . . . . 503

III. Kegel- oder bündelförmiger Krcbs . . . . . . . . 511 
Seite

IV. Knöcherner Krebs . . . . . . . . . . . . . . 514

v. Markschwamm, Zellenkrebs . . . . . . . . . 519

VI. Melanotischer Krebs, Pigmentkrebs, bösartige Melanose - . 528

viI. Gallertkrebs, Alveolarkrebs, Colloidgeschwulst . . . $\quad$. 530

Elftes Capitel. Tuberkeln. . . . . . . . . . . . . . . 535

Anhang zum vierten Abschnitt. Sehmarotzerthiere . . . . . . 541

I. Echinococets hominis. . . . . . . . . . . . . . 541

II. Cyoficercus cellulosae . . . . . . . . . . . . . 546

III. Fllarla medinenols . . . . . . . . . . . . 548 